ANL/TD/CP-92323

CONF-970404 ..

\title{
Materials Effects and Design Implications of Disruptions and Off-Normal Events in ITER
}

\author{
A. Hassanein, \\ Argonne National Laboratory, USA \\ G. Federici, \\ ITER Garching Joint Central Team, Germany \\ I. Konkashbaev, A. Zhitlukhin, \\ Troitsk Institute for Innovation and Fusion Research, Russia \\ V. Litunovsky \\ Efremov Scientific Research Institute, St. Petersburg, Russia
}

RECEIVED

JUN 201997

OSTI

DASTRISUTION OF THIS DOCUMENT is UAMUITED

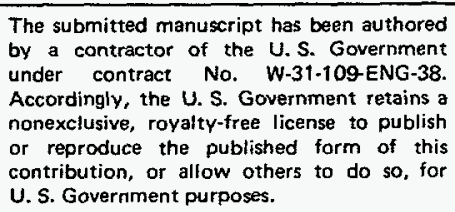

Invited paper presented at the Fourth International Symposium on Fusion Nuclear Technology (ISFNT-4), April 6-11, Tokyo, Japan, 1997. To be published in Fusion Engineering \& Design. 


\section{DISCLAmizR}

Portions of this document may be illegible in electronic irogge produets. Images are produced from the best available original document. 


\section{DISCLAIMER}

This report was prepared as an account of work sponsored by an agency of the United States Government. Neither the United States Government nor any agency thereof, nor any of their employees, make any warranty, express or implied, or assumes any legal liability or responsibility for the accuracy, completeness, or usefulness of any information, apparatus, product, or process disclosed, or represents that its use would not infringe privately owned rights. Reference herein to any specific commercial product, process, or service by trade name, trademark, manufacturer, or otherwise does not necessarily constitute or imply its endorsement, recommendation, or favoring by the United States Government or any agency thereof. The views and opinions of authors expressed herein do not necessarily state or reflect those of the United States Government or any agency thereof. 


\title{
Materials Effects and Design Implications of Disruptions and Off-Normal Events in ITER
}

\author{
A. Hassanein ${ }^{1}$, G. Federici ${ }^{2}$, I. Konkashbaev ${ }^{3}$, V. Litunovsky ${ }^{4}$, \\ and A. Zhitlukhin ${ }^{3}$
}

\author{
${ }^{1}$ Argonne National Laboratory, Argonne, IL 60439, USA \\ ${ }^{2}$ ITER Garching Joint Central Team, D-85748 Garching, Germany \\ ${ }^{3}$ Troitsk Institute for Innovation and Fusion Research, Troitsk, Russia \\ ${ }^{4}$ Efremov Scientific Research Institute, St. Petersburg, Russia
}

\begin{abstract}
Damage to plasma-facing components (PFCs) and structural materials during abnormal plasma behavior such as hard disruptions, edge-localized modes (ELMs), and vertical displacement events (VDEs) is considered a serious life-limiting concern for these components. The PFCs in the International Thermonuclear Experimental Reactor (ITER), such as the divertor, limiter, and parts of the first wall, will be subjected to high energy deposition during these plasma instabilities. High erosion losses on material surfaces, high temperature rise in structural materials (particularly at the bonding interface), and high heat flux levels and possible burnout of the coolant tubes are critical constraints that severely limit component lifetime and therefore degrade reactor performance, safety, and economics. Recently developed computer models and simulation experiments are being used to evaluate various damage to PFCs during the abnormal events. The design implications of plasma-facing and nearby components are discussed, and recommendations are made to mitigate the effects of these events.
\end{abstract}




\section{Introduction}

Damage to plasma-facing components (PFCs) and structural materials due to plasma instabilities in magnetic fusion reactors is one of the most serious concerns for safe, successful, and reliable reactor operation. Plasma instabilities can take various forms such as hard disruptions, which include both thermal and current quench, edge localized modes (ELMs), and vertical displacement events (VDEs). The intense energy deposition (10-200 MJ/m $\left.\mathrm{m}^{2}\right)$ in these events over a short period (0.1-300 ms) may result in severe surface and bulk effects. These include high erosion losses of surface materials, high temperature rise in the structural materials, and high heat flux levels and possible burn-out of coolant tubes.

Recently developed comprehensive models have been enhanced and used for comparison with results of several simulation experiments to analyze and evaluate the thermal response and the erosion damage of ITER PFCs resulting from different plasma instabilities [1,2]. Models for material thermal evolution and phase change, magnetohydrodynamics models for the developed vapor cloud above the surface, and models to calculate resulting radiation, due to vapor heating, and its transport through the vapor cloud are dynamically coupled in a self-consistent approach to evaluate detailed time-dependent responses of plasma-facing materials (PFMs). The extent of the resulting damage to PFMs, structural materials, and coolant channels depends mainly on the total plasma energy deposited, deposition time, and thickness and the type of armor material. During the short disruption events $\left(\tau_{d} \leq 1 \mathrm{~ms}\right)$, initially evaporated material may significantly reduce further PFM vaporization erosion. During longer plasma instabilities, however, such as VDEs ( $\left.\tau_{\mathrm{d}} \approx 100-300 \mathrm{~ms}\right)$ no significant self-shielding is expected to occur and therefore serious erosion and melting can occur. In addition, hydrodynamic 
instabilities and other forces will further erode melt-layers of metallic PFMs. The amount of melt-layer erosion depends on many parameters such as duration of melt layer in the liquid phase, melt-layer thickness, magnitude and direction of various existing forces, and other parameters. Recent experiments on plasma disruption simulation and on melt-layer erosion are evaluated in detail. Longer plasma instabilities may also allow enough time for the deposited plasma energy to be conducted from the surface to the structural material and finally to the coolant channels where it can cause burnout. The analysis has been done for the plasma parameters expected in ITER, for different candidate surface materials such as beryllium, carbon, and tungsten, and for heat sink or structural materials such as copper. The design implications of PFMs and nearby components damage due to these instabilities are discussed and recommendations are made to ensure the best performance and longer lifetime of these components.

\section{Types of damage to PFCs from plasma instabilities}

Plasma instabilities such as hard disruptions, ELMs, and VDEs will cause both surface and bulk damage to plasma-facing and structural materials. Surface damage include high erosion losses due to surface vaporization, spallation, and melt-layer erosion. Bulk damage include large temperature increases in structural materials and at the interface between surface coatings and structural materials. These large temperature increases will cause high thermal stresses, possible melting, and material fatigue and failure. Other bulk effects of some plasma instabilities, particularly those of longer duration such as VDEs and those with deeper deposited energy such as runaway electrons, can cause high heat flux levels at the coolant channels, which may cause burnout of these tubes [3]. 
In addition to these effects, the transport and redeposition of the eroded surface materials from vaporization, melt-layer splashing, and macroscopic particle emission to various locations on plasma-facing and nearby components are of major concern for plasma contamination, for safety (dust inventory hazard), and for successful and prolonged plasma operations following plasma instability events [4]. A procedure to clean up the dust and redeposited debris of the eroded materials is just as important as the procedure to repair the incurred damage on PFCs.

\section{Surface erosion mechanisms}

Models for surface vaporization, material cracking and spallation, and liquid-metal ejection of melt layers have been developed for various erosion-causing mechanisms and implemented in the comprehensive A*THERMAL-S and SPLASH computer codes [5-7]. Below are brief descriptions of various models and mechanisms used to study surface erosion and predict components' lifetimes.

\subsection{Erosion due to surface vaporization}

To evaluate the early response of PFCs to plasma instabilities, the detailed physics of various interaction stages of plasma particles with target materials must be correctly modeled in detail. Initially, the incident plasma particles of the disrupting plasma will deposit their energy at the surface of the target material. Models for particle energy deposition and material thermal evolution were developed for multilayer structures which include phase-change and surface-vaporization models, moving boundaries, temperaturedependent thermophysical properties, etc. Deposition at higher power will cause sudden and early surface ablation of PFCs. As a result, a vapor cloud of the surface material will 
quickly form above the bombarded surface and in front of the incoming plasma particles. Depending on many parameters, such as incident plasma power, magnetic field structure, geometrical considerations, vapor diffusion and motion, etc., the developed vapor cloud may significantly shield the original exposed areas from the incoming plasma particles and therefore further reduce surface damage.

To calculate the efficiency of vapor-cloud shielding in protecting plasma-facing materials (PFMs), detailed physics of plasma/vapor interactions have been modeled. The models include plasma particle slowdown and energy deposition in the expanding vapor, vapor heating, excitation, and ionization, and vapor-generated proton radiation. The detailed vapor motion above the exposed surface is calculated by solving the vapor magnetohydrodynamic equations for conservation of mass, momentum, and energy under the influence of a strong magnetic field [2]. A significant part of the incident initial plasma kinetic energy is quickly transformed into vapor-generated photon radiation.

Finally, models for photon transport throughout the expanding vapor cloud have been developed to calculate the net heat flux that is reaching the original disruption surface of PFCs [7]. It is this heat flux that will further determine most of the response and the net erosion from surface vaporization of PFCs during these instabilities. The models include treatment for nonlocal thermodynamic equilibrium of the vapor-cloud-generated plasma, multigroup analysis (up to 4000 energy groups) of the produced photon spectra, as well as self-consistent kinetic models for both continuum and line radiation that also take into account treatment of multidimensional radiation transport, and advanced numerical techniques for accurate calculation of radiation propagation in the vapor cloud as well as in the condensed target material. 
The energy density deposited on the divertor surface during a disruption can range from 10 to $200 \mathrm{MJ} / \mathrm{m}^{2}$. Figure 1 shows tungsten solid/liquid/vapor temperatures as a function of distance for an incident plasma energy density of $10 \mathrm{MJ} / \mathrm{m}^{2}$ deposited in disruption times of 0.1 and $1 \mathrm{~ms}$. A magnetic field strength of $5 \mathrm{~T}$ with an incident angle of $2^{\circ}$ is assumed in this analysis. At the shorter disruption, both the liquid/solid surface and the vapor temperatures are higher than at the longer disruption. A longer disruption time causes the vapor to expand to greater distances above the divertor surface and also causes the energy flux at the divertor surface to diffuse deeper into the bulk and produce a thicker melt layer. The temperature of the hot region of the vapor-cloud generated plasma can range from a few $\mathrm{eV}$ up to few tens of $\mathrm{eV}$, depending on the target material and on the incident plasma power density and particle kinetic energy. Significant radiation can be emitted from the hot vapor-front zones. In fact, in most high-energy disruption cases, more than $80 \%$ of the incident plasma energy is converted to photon radiation. Depending on divertor design and configuration, the expanding hot vapor and its radiation can damage nearby components [8], particularly in closed divertor configurations [9]. It is therefore desirable that the vapor normal expansion be kept to a minimum and that the design of the divertor region not be of the closed type, in order to allow the larger surface area to absorb the intense emitted radiation.

The time evolution of the transmitted power to the tungsten surface and the thickness of the resulting vaporization during disruptions at two energy densities (10 and $100 \mathrm{MJ} / \mathrm{m}^{2}$ ) are shown in Fig. 2. The power transmitted to the original surface is quickly and significantly reduced as soon as surface vapor begins to accumulate and form a cloud above the exposed surface area. This process effectively shields the surface from the direct deposition of plasma particles. Because of the relatively low kinetic energy of the incident plasma particles $(\mathrm{E}<20 \mathrm{keV})$, very little vapor is needed to completely stop incoming particles, particularly when the vapor is heated [10]. The total erosion depth due 
to surface vaporization is only a few micrometers, which is about one to two orders of magnitude lower than it would be if no vapor shielding was assumed [11]. Figure 2 also shows that increasing the power density by one order of magnitude increases vaporization losses by a factor of only about 2 because, at higher incident plasma energy densities, the transmitted energy flux to the surface is only slightly higher, mainly due to the higher photon flux generated in the vapor cloud. This higher energy flux that is transmitted to the tungsten surface will slightly increase the surface temperature and generate more vapor, which then becomes more opaque to the incoming higher radiation flux.

To compare erosion losses derived from only surface vaporization models, recent experimental data, from plasma gun devices, is analyzed using carbon-based materials (CBMs), which do not melt. Despite the large uncertainties in the parameters of plasma gun devices, they are believed to be more suitable for simulating reactor disruptions than are electron or laser beams [7]. Several laboratory experiments were performed to study material erosion in plasma gun facilities such as the PLADIS [12] at Sandia, the QSPA [13] at TRINITI, and the VIKA [14] device at the Efremov Institute. Most of these facilities can generate a low-temperature plasma $(\mathrm{T}<1 \mathrm{keV})$ with high energy flow of up to $30 \mathrm{MJ} / \mathrm{m}^{2}$ deposited in a pulsed duration of $<1 \mathrm{~ms}$. Figure 3 shows the calculated erosion rates of two different CBMs (POCO and MPG-8 graphite), from surface vaporization as a function of incident plasma energy density during the simulated disruption, in comparison with experimental results from the PLADIS and VIKA plasma gun devices. The dashed lines among the different sets of data are for visual orientation. The differences between the two plasma gun facilities may be due to uncertainties in the deposited energy and diagnostic methods, sample type and composition, and plasma-flow parameters. Agreement with model predictions is better at lower energy densities. Other experimental data from laser, electron beam, and plasma gun devices have shown much higher graphite erosion rates. Models for other than surface vaporization are being developed to predict explosive and brittle-destruction erosion of graphite [15]. The carbon surface vaporization rate is slightly 
higher than the metallic surface vaporization of beryllium and tungsten, as determined by the complex interplay of various material properties and thermodynamics, vapor magnetohydrodynamics, and detailed photon radiation transport in vapor-cloud plasma [6]. However, because CBMs do not melt and can have larger initial thickness, they may have much longer lifetimes and/or fewer down-times for repair than those of metallic components.

\subsection{Melt-layer erosion mechanisms}

Theoretical calculations have already shown that surface vaporization losses of metallic PFMs are small (only a few micrometers deep) over a wide range of plasma conditions during the shorter plasma instabilities (such as the hard disruptions shown in Fig 2). This is due to the self-shielding mechanism discussed above in which the material's own vapor stops and absorbs most of the incoming plasma energy. The net energy flux to the original disruption area is significantly reduced to $<5-10 \%$ of its initial value (see Fig. 2), depending on target material and the initial plasma energy flux. This reduced energy flux is, however, large enough to cause significant melting of metallic PFCs. The resulting melt-layer thickness can be one to two orders of magnitude higher than surface vaporization losses [6]. The free surface of the melt layer, developed during a disruption, is exposed to various forces such as electromagnetism, gravitation, mechanical vibration, plasma momentum, surface tension, and ablation recoil. Several mechanisms can cause melt-layer loss during the thermal quench phase of the disruption [16]. These include melt splashing from boiling of gas bubbles, splashing due to absorption of plasma momentum, erosion from hydrodynamic instabilities developed in the liquid layer due to the tangential and perpendicular forces acting on the free surface of the liquid, erosion due to runoff of melt layers over the structure, and erosion caused by mechanical vibration of the machine during the disruption event. 
Among the different mechanisms that can cause melt-layer erosion during plasma instabilities, two have been demonstrated experimentally and studied theoretically in detail $[17,18]$. Contributing significantly to melt layer erosion at high heat loads is melt splashing due to the formation, growth, and bursting of bubbles inside the liquid layer. This results from the continuous heating and overheating of the liquid layer during energy deposition. Surface temperature of the liquid layer will exceed the equilibrium vaporization temperature for periods of time during plasma/material interaction, and this overheating will result in the growth and the explosion or vaporization of the volume bubbles as they reach the free surface, in turn leading to ejection and loss of parts of the melt layer. The amount and rate of melt-layer erosion depend on many parameters, such as degree of overheating, impurity and gas content, material properties, and disrupting plasma parameters. Preliminary analysis of the microstructure of the exposed metal surfaces in the QSPA facility has clearly shown the formation of volume bubbles at high densities [13]. This was also shown for other materials such as $\mathrm{B}_{4} \mathrm{C}$ in the VIKA plasma gun [19] and a tungsten sample in the GOL-3 electron beam facility [20]. Traces of melted aluminum metal droplets were found at locations in the QSPA facility up to a few meters from the aluminum target area.

A second mechanism in melt-layer erosion, also demonstrated experimentally, is the development and growth of hydrodynamic instabilities. Such forces can occur during the thermal and current quench phases of a disruption because of plasma impact momentum (plasma wind) at the liquid surface and from forces generated due to current decay in the liquid metal layer. During the thermal quench, part of the incident plasma momentum is deposited in a thin surface layer; this will accelerate the liquid metal in this layer to very high velocities. As a result, hydrodynamic instabilities such as the Kelvin-Helmholtz (KH) instability will arise and form liquid droplets that will be carried away by the plasma 
wind. Careful analysis of irradiated surfaces has demonstrated that $\mathrm{K}-\mathrm{H}$ instability may exist and act as a melt-layer erosion mechanism. Near the central areas of the irradiated targets, where the velocity of the incident plasma stream along the sample surface is close to zero, one can clearly see the volume bubbles. Near and outside the sample peripheral areas, however, one can see liquid droplets with long tracks formed because of the high plasma stream velocity; this may suggest the existence of $\mathrm{K}-\mathrm{H}$ instability [13]. These droplets will be transported by the plasma wind, whose highest velocities are along the edges of the sample surface because of the changing plasma flow around the more intense central vapor cloud.

\subsection{Theoretical predictions of melt-layer erosion}

Improved numerical models have been implemented in detail in the SPLASH code to study dynamic erosion of the evolving melt layer due to various mechanisms and different existing forces. This code is coupled with the A*THERMAL-S code, which calculates the details of plasma/vapor interaction, in order to accurately predict melt-layer evolution, time-dependent melt erosion, and the interaction with the developing vapor cloud above the liquid surface during intense energy deposition. The analysis presented in this work is devoted to the study of melt-layer erosion due to bubble vaporization and $\mathrm{K}-\mathrm{H}$ hydrodynamic instability to help explain the experimental results.

To correctly simulate melt-layer erosion, a four-moving-boundaries problem is solved [18]. The front of the vapor cloud, generated from the initial plasma energy deposition, is one moving boundary determined from the solution of vapor hydrodynamic equations. The second moving boundary due to surface vaporization of the target is calculated from target thermodynamics. Immediately following the surface vaporization front is a third moving boundary due to the melt layer splashing front. Finally, the fourth 
moving boundary is the liquid/solid interface, which further determines the new thickness of the melt layer. These moving boundaries are interdependent, and a self-consistent solution must link them dynamically and simultaneously. It is the third moving boundary (the liquid splashing front), however, that determines the extent of metallic PFC erosion and lifetime due to plasma instabilities.

Additional laboratory experiments in the plasma gun facilities of PLADIS, QSPA, and the VIKA device were also performed to study melt-layer erosion. Most results from these facilities have demonstrated significant losses and erosion of the developed melt layers of metallic targets. Figure 4 shows the eroded thickness of aluminum targets derived from mass-loss measurements as a function of the incident plasma energy density at these plasma gun facilities, in comparison to model predictions from the A*THERMAL-S and SPLASH computer codes. Also shown is the only Be-eroded depth data obtained from the PLADIS plasma gun at $100 \mu$ s [21]. The mass-loss measurements usually yield an erosion depth that is shallower by a factor of about 3 to 5 than the maximum depth recorded by surface profilometry, due to factors such as gun beam profile, melt-layer movement, and possible redeposited material (particularly near sample edges) [14]. At high plasma energy densities $\left(>10 \mathrm{MJ} / \mathrm{m}^{2}\right)$, the average eroded thickness is much higher than the predicted thickness from only surface vaporization. In fact, at the higher energies the eroded thickness can significantly exceed the sum of both the calculated surface vaporization and the stationary melt layer thickness and in most cases the net thickness eroded is more than two to three times that of the stationary melt-layer thickness. The stationary thickness is defined as the resulting melt-layer depth if no movement of the melt layer is assumed. This implies that the evolving melt layer is lost while it is being developed. Other plasma gun facilities have yielded even higher aluminum erosion thicknesses $(>200 \mu \mathrm{m})$ at a much lower energy density $\left(E \approx 3 \cdot 3 \mathrm{MJ} / \mathrm{m}^{2}\right)$ deposited within $100 \mu \mathrm{s}[22]$. Such differences 
among various plasma gun facilities can be attributed to the many uncertainties in energy calibration and diagnostic methods, mass loss vs. surface profilometry of exposed samples, sample geometry, the incident plasma dynamic pressure, and plasma particle kinetic energy.

The models of melt-layer erosion mechanisms are generally in good agreement with the experimental data but slightly underestimate the average eroded depth at the higher energy densities of the QSPA facility. This may suggest additional erosion mechanisms such as that due to nonuniform incident plasma dynamic pressure [14]. Another possible melt-layer erosion mechanism, implemented in the SPLASH code but not included in this study, is Raleigh-Taylor hydrodynamic instability due to inertial forces from the acceleration of the melt front at the solid/liquid interface. The relative contributions of the various erosion mechanisms depend on the magnitude of incident plasma momentum, degree of liquid overheating, liquid metal properties, geometrical effects, etc. Melt-layer erosion of heavier materials such as copper, for example, is lower by a factor of $\approx 3$ than aluminum erosion under similar irradiation conditions of the QSPA facility. This is explained by the modeling simulation as having two main causes. The first is the required higher energy to remove the volume bubbles and the associated heavier liquid droplets. The second is the lower splashing velocity from $\mathrm{K}-\mathrm{H}$ instability, again because of the higher density effect that requires more energy to liberate the liquid droplets. Melt-layer erosion of much heavier materials such as tungsten, however, was shown to be very low in plasma gun experiments [14,21]. This may have several causes, such as the much higher fraction of the incident plasma-energy reflected from the heavier target surface due to the very low kinetic energy of incident plasma particles.

The anticipated disruption lifetime performance of a metallic PFM such as beryllium is shown in Fig. 5. The maximum number of tolerated disruptions is shown as a function 
of the disruption time for incident plasma energy density of $10 \mathrm{MJ} / \mathrm{m}^{2}$. The initial thickness of the beryllium component is assumed to be $5 \mathrm{~mm}$ of which $50 \%$ is assumed to be sacrificed to disruption erosion. The lifetime is shown for erosion losses due to vaporization only, vaporization and melting, and vaporization with twice the stationary melting thickness. Tungsten disruption lifetime is somewhat similar to that of beryllium. Larger initial thickness of PFMs will increase erosion lifetime. The maximum initial thickness is, however, limited by the maximum allowable surface temperature which depends on normal operating conditions as well as material constraints. Longer disruption times and higher energy densities usually result in thicker melt layers. This could even lead to further reduction in disruption lifetime. Significant losses of melt layers can reduce PFC disruption lifetime to only a few disruptions. This clearly shows the significant disadvantage of using metallic components for plasma-facing and nearby components. Therefore, metallic PFCs are not recommended at or near locations where intense and frequent disruptions may occur. However, damaged metallic PFCs are easier to repair in situ and are more resistant to neutron damage. Further analyses are required for more accurate assessment of melt-layer erosion in ITER-relevant conditions.

\section{Effects of longer plasma instabilities}

Although thermal-quench disruptions have no significant thermal effects on structural materials and coolant channels, VDEs, in addition to causing severe surface melting and erosion, can result in substantial damage to these components [3]. Because of the longer deposition time of VDEs (100-300 ms), much of the plasma incident energy will be conducted through the surface coating material onto the copper structural material and finally to the coolant channels. One concern is the higher temperature observed in the structural material, particularly at the interface with the coating materials. Higher temperatures cause high thermal stresses in the structure and seriously degrade the integrity 
of the interface bonding because of thermal diffusion and formation of intermetallic compounds, which may lead to detachment of the coating from the structural material. Figure 6 shows the temperature of a copper surface at its interface with tungsten or beryllium coatings of $5 \mathrm{~mm}$ thickness or with 20 -mm-thick carbon tiles over the $5 \mathrm{~mm}$ copper substrate during a typical VDE. Surface coating and tile thickness are determined by the surface temperature limitations during normal operation. For ITER conditions, the thickness of beryllium or tungsten divertor targets is usually $<10 \mathrm{~mm}$. Thinner coatings are also desirable because of cost, safety, and concern about plasma contamination. Tungsten and carbon coatings of similar thicknesses usually result in similar and higher copper surface temperature than that of a beryllium coating because most of the incident plasma energy is removed by the beryllium's higher surface vaporization rate, which leaves little energy to be conducted through the structural material [3]. In the case of a 5-mm-thick tungsten coating, the copper surface interface actually melts. Only beryllium coatings of reasonable thickness $(<5-10 \mathrm{~mm})$ or very thick carbon tiles $(>20 \mathrm{~mm})$ can withstand the acceptable temperature rise in the copper structure for the conditions shown.

The heat conducted through the structural material will eventually reach the coolant channels. Figure 7 shows heat flux to the water coolant from beryllium, tungsten, and carbon coatings and tiles on the copper structure. Similar results to this work were also predicted [23]. Again, in the case of tungsten, the heat flux is so high that it can exceed the critical value and last up to a few seconds, causing burnout of the coolant channels.

Carbon tile thickness of $20 \mathrm{~mm}$ or more will reduce both the interface copper surface temperature and the heat flux to the coolant to acceptable limits. The reason for this reduction is that thicker coatings usually result in higher surface temperatures before and during VDEs and disruptions that may be acceptable for CBMs. This tends to increase surface erosion from vaporization during these events. Therefore, more incident plasma 
energy is removed by the hotter surface, leaving less incident energy to be conducted to the structure and to the coolant over a longer period because of the thicker coating. In these calculations, the initial value of the heat flux during normal operation, prior to VDEs and disruptions, is assumed in these calculations to be $5 \mathrm{MW} / \mathrm{m}^{2}$. A VDE may also occur on the limiter surface prior to normal operations, i.e., during start-up with lower heat flux values. This will slightly reduce the effects of VDEs in erosion of surface-coating materials, temperature rise in structural materials, and heat fluxes to coolant channels [3].

\section{Conclusions}

Various effects of plasma/material interactions during plasma instabilities such as disruptions and VDEs have been studied with a comprehensive dynamic model that integrates in fine detail the structure's thermal evolution, physics of plasma/vapor interactions, magnetohydrodynamics, and photon radiation transport of multilayer structures. Various plasma instabilities result in different damage to PFMs and structural materials. Theoretical predictions of A*THERMAL-S and SPLASH codes are generally in good agreement with the experimental results. Carbon-based materials may be preferred to metallic materials in PFCs, from the standpoint of disruption-caused erosion. Thicker carbon tiles may also be favored as a PFM to reduce VDE erosion and plasma contamination and to mitigate various thermal effects because carbon is a lower-atomicnumber material and does not melt. However, carbon has several serious disadvantages, such as high explosive erosion at high energy densities, large tritium inventory due to codeposition, high chemical sputtering, radiation-enhanced sublimation, and low resistance to neutron damage. More-detailed modeling and more reactor-relevant simulation experiments are required before a final recommendation is made toward the selection of PFMs. In general, plasma instabilities must be avoided or sharply minimized. In addition, 
the effects of redeposited debris from the eroded materials on plasma contamination and on subsequent reactor operations must be studied further.

\section{Acknowledgments}

This work is supported by the U.S. Department of Energy, Office of Fusion Energy, and by the Ministry of Atomic Energy and Industry, Russia.

\section{References}

[1] A. Hassanein and I. Konkashbaev, J. Nucl. Mater. 220-222 (1995) 244.

[2] A. Hassanein and I. Konkashbaev, Fusion Eng. Des. 28 (1995) 27.

[3] A. Hassanein, Fusion Technol. 30, No. 3, Part 2A (1996) 713.

[4] A. Hassanein, Fusion Technol. 19, Number 3, PART 2B, (1991) 1789.

[5] A. Hassanein and I. Konkashbaev, Suppl. J. Nucl. Fusion 5 (1994) 193.

[6] A. Hassanein and I. Konkashbaev, J. Nucl. Mater. 233-237 (1996) 713.

[7] A. Hassanein, Fusion Technol. 26 (1994) 532. 
[8] A. Hassanein and I. Konkashbaev, "Erosion damage of nearby plasma-facing components during a disruption on the divertor plate," Proc. of 19th Symp. on Fusion Technology, Sept. 16-20, 1996, Lisbon, Portugal.

[9] G. Janeschitz et al., J. Nucl. Mater. 220-222 (1995) 73.

[10] T. A. Mehlhorn, "A finite material temperature model for ion energy deposition in ion-driven ICF targets," SAND 80-0038 (1980).

[11] A. Hassanein, "Response of Materials to High Heat Fluxes during Operation in Fusion Reactors," ASME, 88-WA/NE-2, (1988).

[12] J. Gahl et al., J. Nucl. Mater. 191-194 (1992) 454.

[13] V. Belan et al., J. Nucl. Mater. 233-237 (1996) 763.

[14] V. Litunovsky et al., 16th IEEE/NPSS Symp. on Fusion Engineering, Sept. 30-Oct. - 5 , (1995) 435 .

[15] A. Hassanein et al., to be published in Plasma Devices and Operations, 1997.

[16] A. Hassanein, Fusion Technol. 15 (1989) 513.

[17] A. Hassanein et al., Fusion Technol. 1 (1994) 223. 
[18] A. Hassanein et al.," Modeling and simulation of melt-layer erosion during a plasma disruption," Proc. of 12th Int. Conf. on Plasma Surface Interactions in Controlled Fusion Devices, Saint-Raphael, France, May 20-24,1996, to be published in J. Nucl. Mater.

[19] T. Burtseva et al., Plasma Devices and Operations, Vol. 4 (1995) 31.

[20] A. Burdakov et al., J. Nucl. Mater. 233-237 (1996) 697.

[21] A. Hassanein, D. Ehst, and J. Gahl, J. Nucl. Mater. 212-215 (1994) 1272.

[22] J. Gilligan et al., J. Nucl. Mater. 196-198 (1992) 596.

[23] G. Federici and A. Raffray, J. Nucl. Mater. 244 (1997) 101. 


\section{Figure Captions}

Figure 1 Tungsten target and vapor temperatures as a function of distance during a disruption.

Figure 2 Power transmitted to tungsten surface, and resulting vaporization thickness during a disruption.

Figure 3 Experimental data and current vaporization models for carbon-based materials.

Figure 4 Aluminum erosion depth in different gun experiments and current model predictions; beryllium depth from PLADIS plasma gun also shown.

Figure 5 Maximum tolerated number of disruptions in beryllium, based on models and experimental results.

Figure 6 Rise in copper surface temperature of carbon-, beryllium-, and tungstencoated copper substrates during a VDE.

Figure 7 Heat flux to coolant of carbon-, beryllium-, or tungsten-coated copper structure during a VDE. 


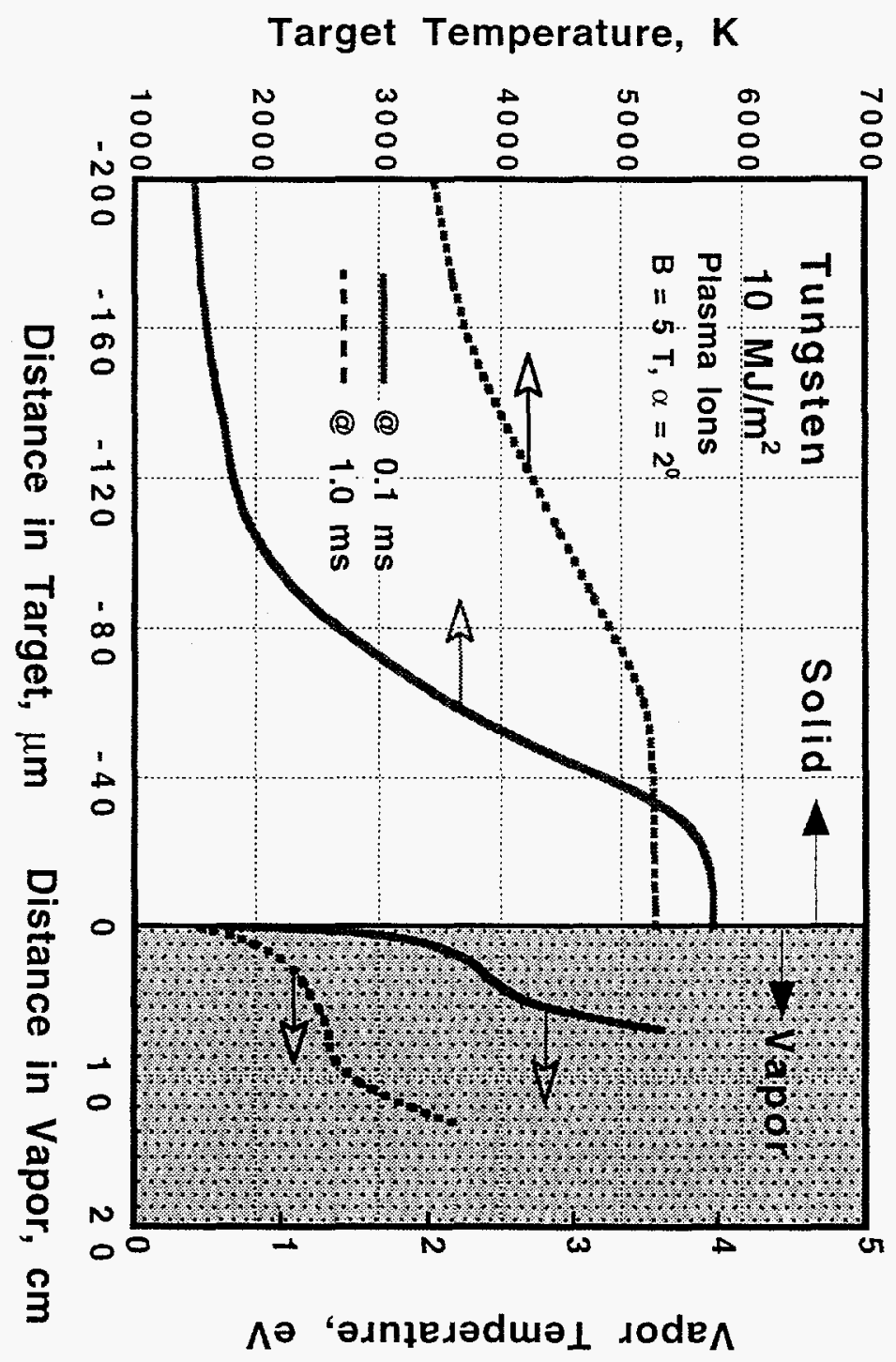




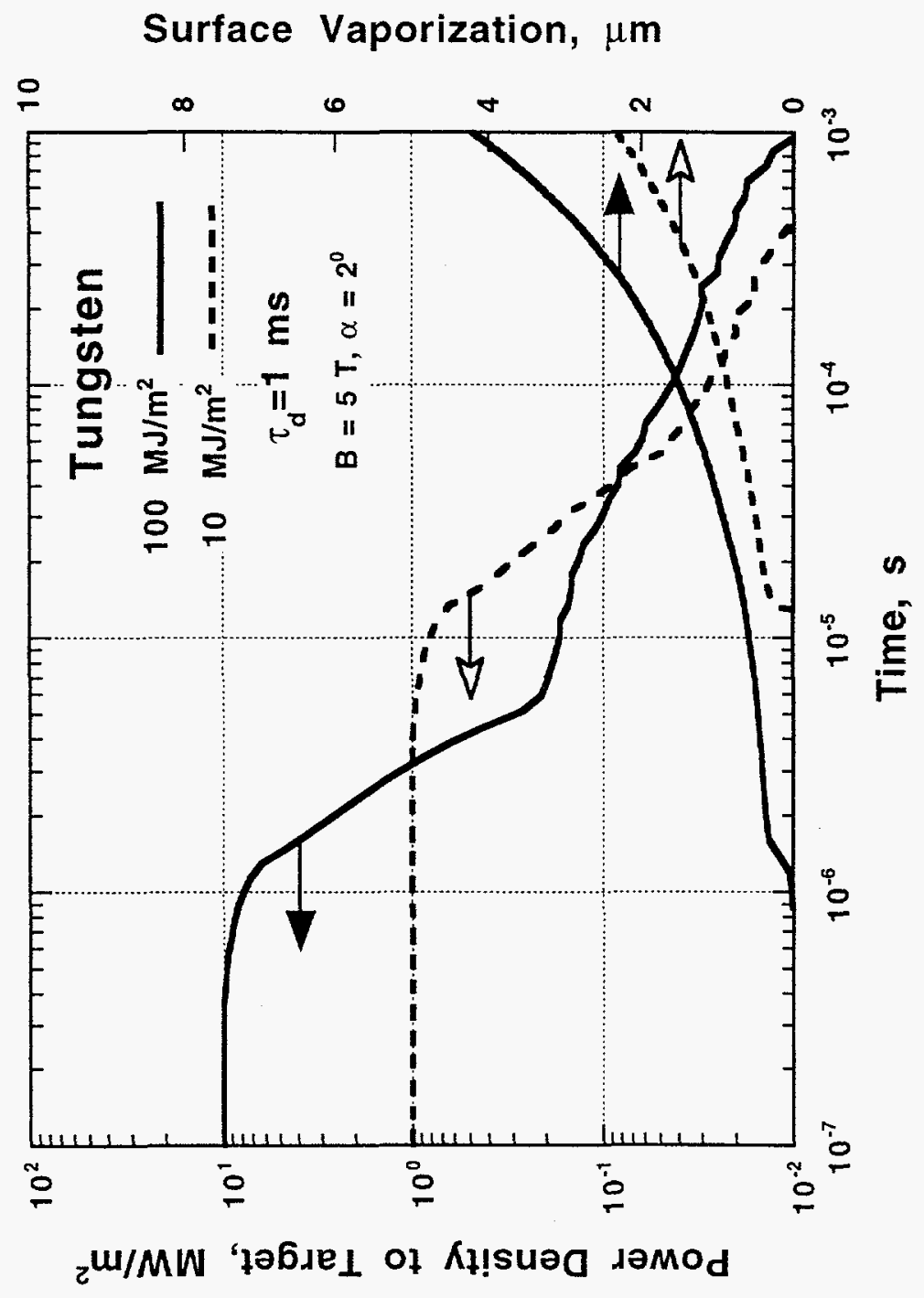




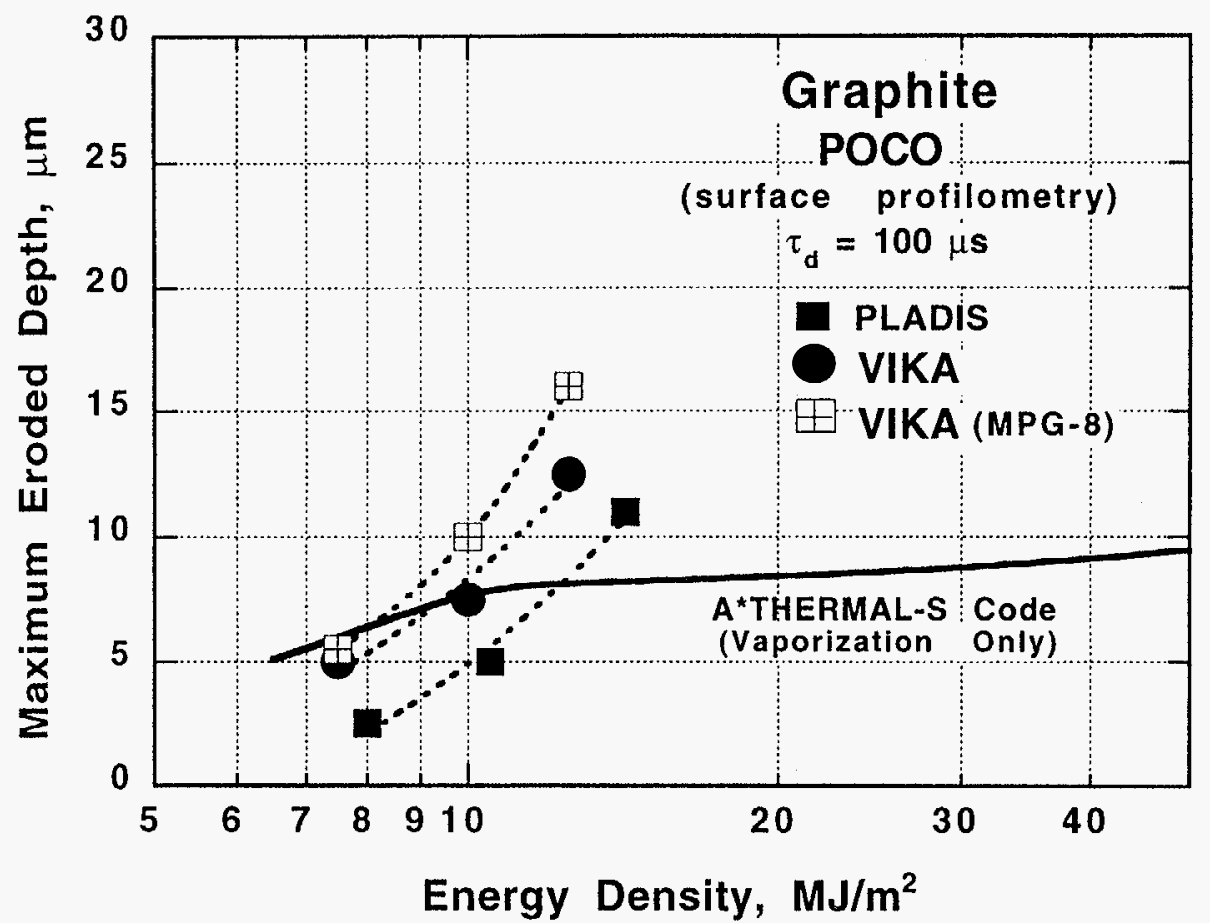




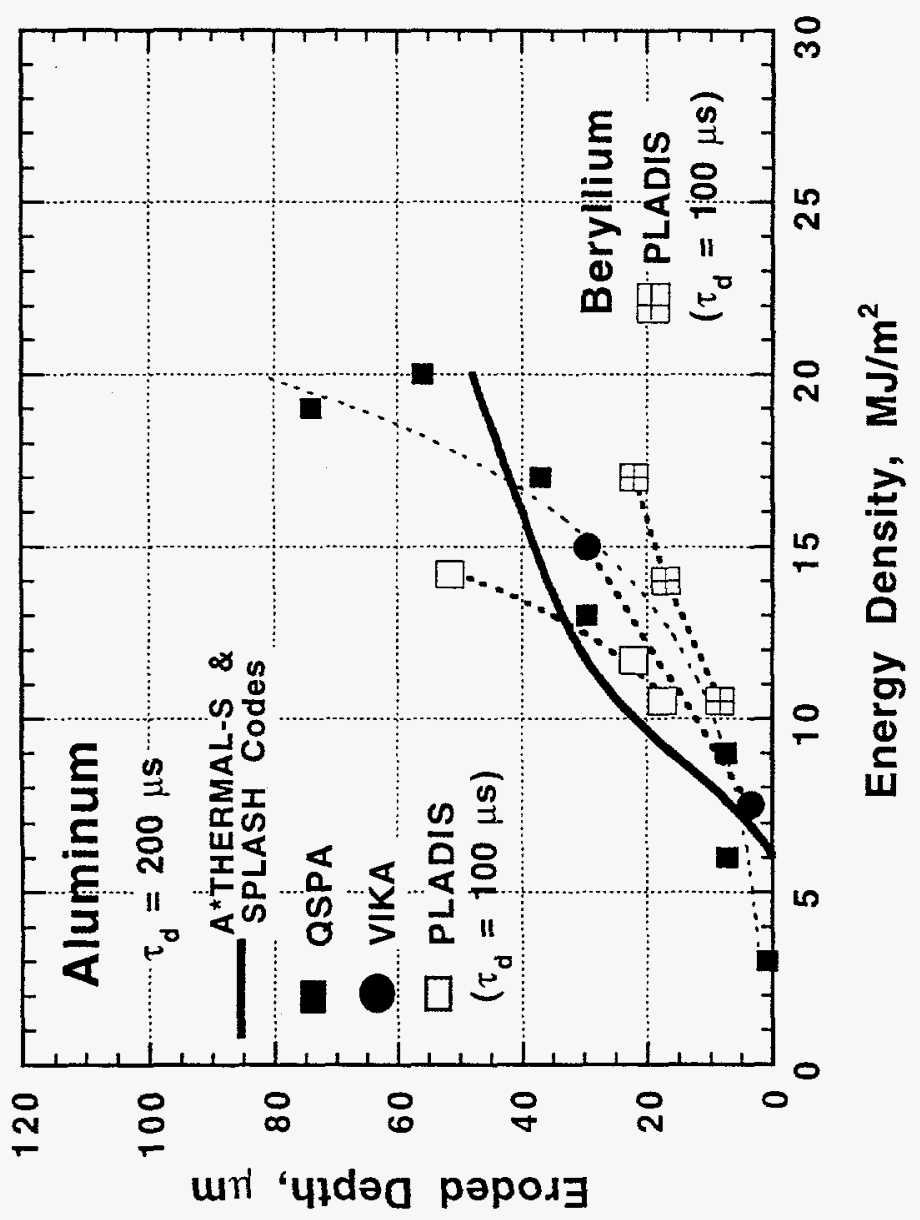




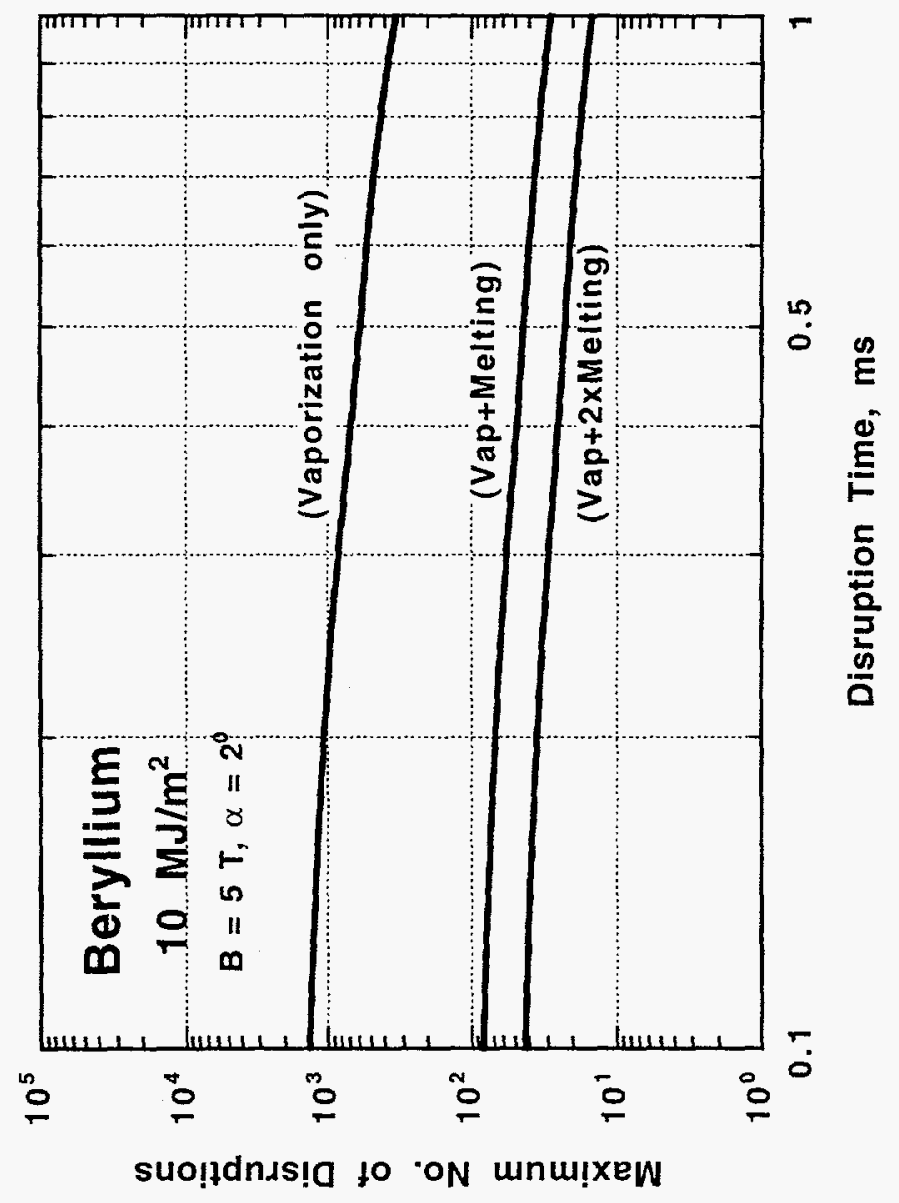




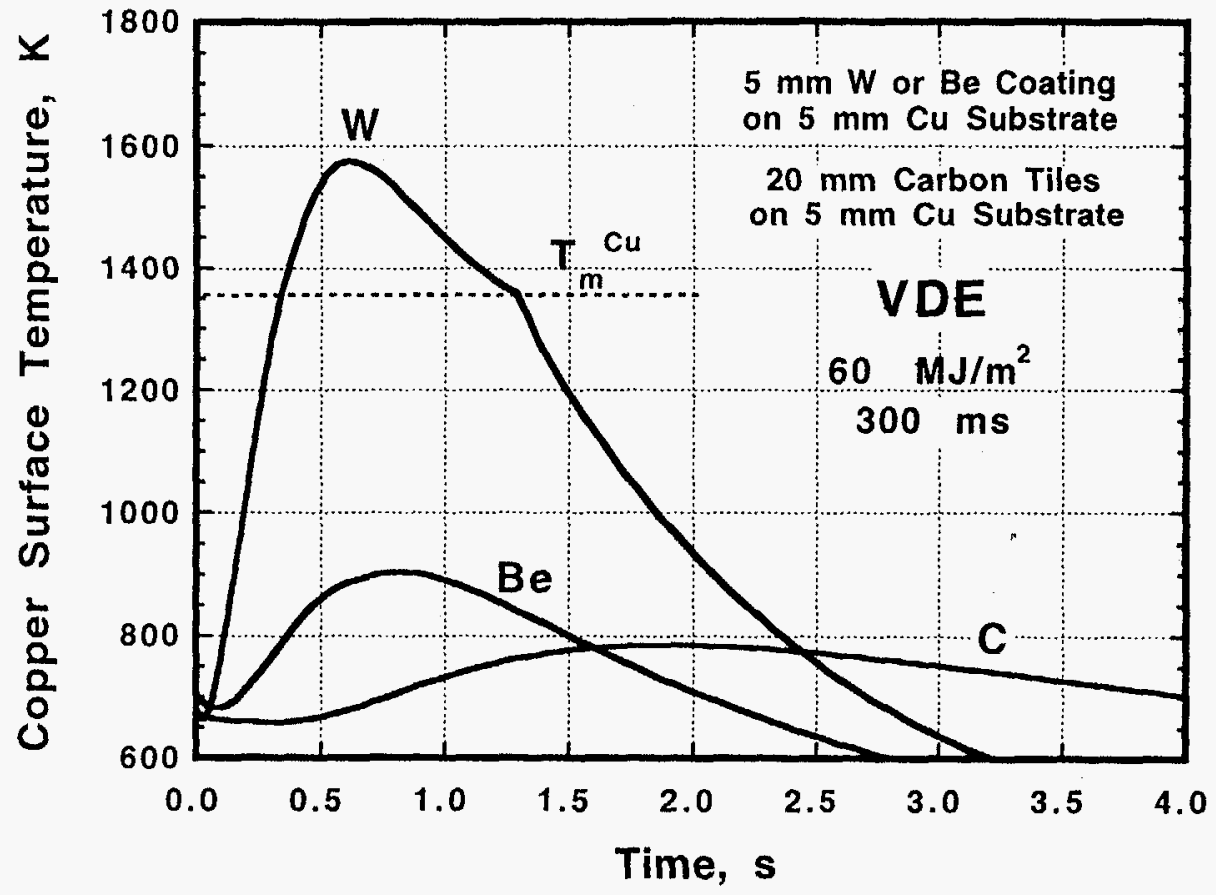




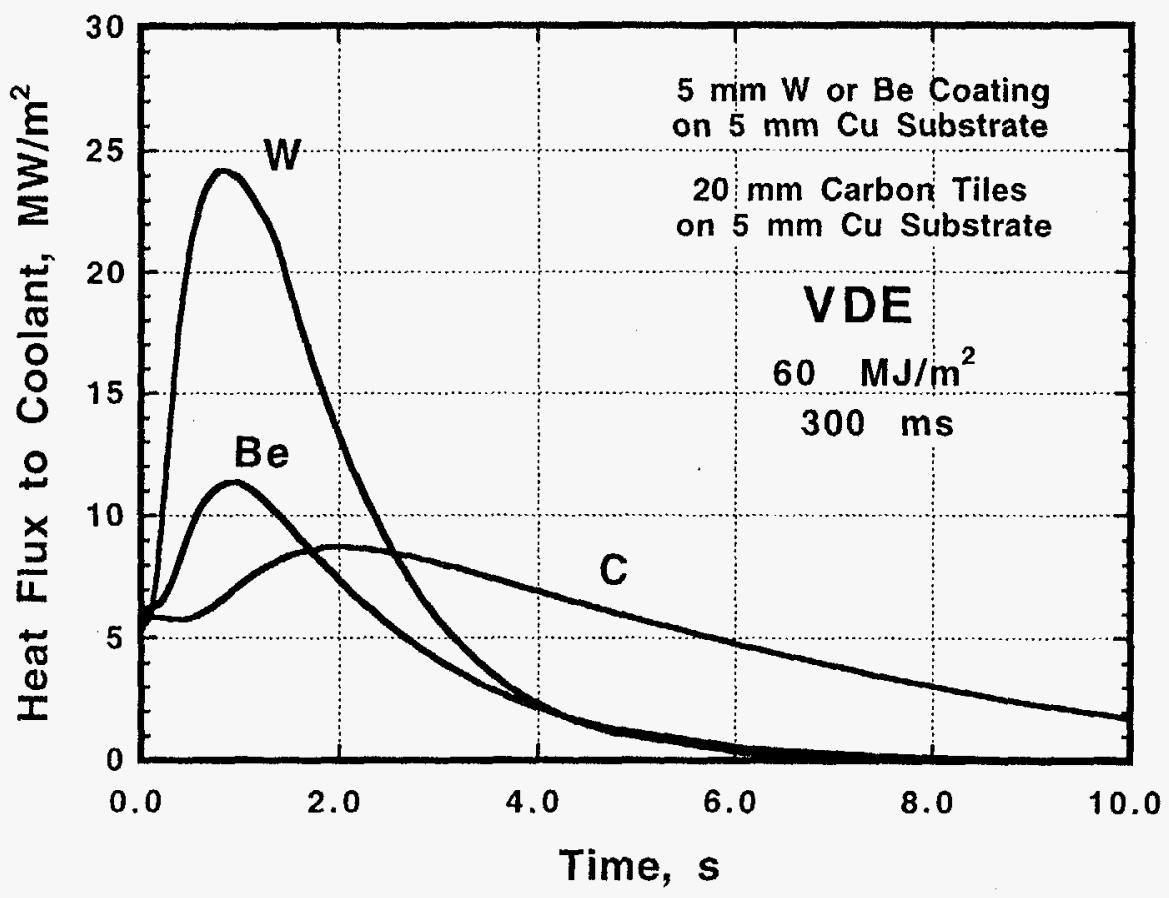

